\title{
Histone H2A monoubiquitylation and p38-MAPKs regulate immediate-early gene-like reactivation of latent retrovirus HTLV-1
}

\author{
Anurag Kulkarni, ${ }^{1}$ Graham P. Taylor, ${ }^{1}$ Robert J. Klose, ${ }^{2}$ Christopher J. Schofield, ${ }^{3}$ \\ and Charles R.M. Bangham¹ \\ 'Division of Infectious Diseases, Department of Medicine, Imperial College, London, United Kingdom. 'Laboratory of \\ Chromatin Biology and Transcription, Department of Biochemistry, and ${ }^{3}$ Chemistry Research Laboratory, Department of \\ Chemistry, University of Oxford, Oxford, United Kingdom.
}

It is not understood how the human T cell leukemia virus human T-lymphotropic virus-1 (HTLV-1), a retrovirus, regulates the in vivo balance between transcriptional latency and reactivation. The HTLV-1 proviral plus-strand is typically transcriptionally silent in freshly isolated peripheral blood mononuclear cells from infected individuals, but after short-term ex vivo culture, there is a strong, spontaneous burst of proviral plus-strand transcription. Here, we demonstrate that proviral reactivation in freshly isolated, naturally infected primary $C D 4^{+} \mathrm{T}$ cells has 3 key attributes characteristic of an immediate-early gene. Plus-strand transcription is p38-MAPK dependent and is not inhibited by protein synthesis inhibitors. Ubiquitylation of histone H2A (H2AK119ub1), a signature of polycomb repressive complex-1 (PRC1), is enriched at the latent HTLV-1 provirus, and immediate-early proviral reactivation is associated with rapid deubiquitylation of $\mathrm{H} 2 \mathrm{~A}$ at the provirus. Inhibition of deubiquitylation by the deubiquitinase (DUB) inhibitor PR619 reverses H2AK119ub1 depletion and strongly inhibits plus-strand transcription. We conclude that the HTLV-1 proviral plus-strand is regulated with characteristics of a cellular immediate-early gene, with a PRC1-dependent bivalent promoter sensitive to p38-MAPK signaling. Finally, we compare the epigenetic signatures of p38-MAPK inhibition, DUB inhibition, and glucose deprivation at the HTLV-1 provirus, and we show that these pathways act as independent checkpoints regulating proviral reactivation from latency.

License: This work is licensed under the Creative Commons Attribution 4.0 International License. To view a copy of this license, visit http:// creativecommons.org/licenses/ by/4.0\%.

Conflict of interest: The authors have declared that no conflict of interest exists.

License: Copyright 2018, American Society for Clinical Investigation.

Submitted: June 26, 2018

Accepted: August 30, 2018

Published: October 18, 2018

\section{Reference information:} JCI Insight. 2018;3(20):e123196. https://doi.org/10.1172/jci. insight.123196.

\section{Introduction}

Human T-lymphotropic virus-1 (HTLV-1), also known as the human T cell leukemia virus, is a retrovirus that infects over 10 million individuals worldwide. The infection is asymptomatic in most cases. However, between $5 \%$ and $10 \%$ of infected individuals develop either a debilitating neuroinflammatory condition called HTLV-1-associated myelopathy (HAM) or a refractory T cell neoplasm with a poor prognosis, adult T cell lymphoma/leukemia (ATLL) $(1,2)$.

The $9 \mathrm{~kb}$ HTLV-1 provirus is flanked by 2 identical long terminal repeat (LTR) sequences, which contain directional promoters that govern transcription of the proviral plus- and minus-strands. The virus spreads within the host either through transfer of infectious virions from cell to cell (infectious spread) via the virological synapse, or through duplication of the provirus together with the host cell genome during cell division (mitotic spread) (3). During infectious spread, the first HTLV-1 transcript encodes the transactivator of viral transcription (Tax). Tax initiates a positive feedback loop by further activating the 5'-LTR promoter that governs HTLV-1 plus-strand transcription, thereby stimulating productive viral replication and virion production $(4,5)$. Infectious spread predominates during acute infection, when the percentage of infected cells (the proviral load) in the host is low. The relative contribution of mitotic spread to proviral load is greater during the chronic phase of infection (6). In a typical infected individual, there are between $1 \times 10^{4}$ and $1 \times 10^{5}$ clones of infected cells, each with a unique proviral integration site in the host genome (6). 
HTLV-1 appears to be mainly latent during chronic infection because there are usually no detectable viral structural RNA or protein products in freshly isolated infected cells (7-9). However, the presence of a chronically activated cytotoxic T cell response to HTLV-1 plus-strand-encoded antigens in infected individuals suggests that proviral expression continues constantly, albeit at a low level $(10,11)$. We therefore hypothesized that the HTLV-1 proviral plus-strand is expressed in intermittent bursts; recent evidence from single-molecule RNA FISH (smFISH) (12) and real-time imaging (13) supports this hypothesis. These observations raise the question, what regulates the reactivation and silencing of the HTLV-1 provirus? To investigate this question, we exploited a long-standing observation: whereas plus-strand transcription is usually undetectable in freshly isolated infected peripheral blood mononuclear cells (PBMCs), there is a sudden spurt in plus-strand proviral gene transcription after a short period of ex vivo culture (7-9).

It is known that storage of blood at ambient temperature for even 1 hour causes upregulation of a number of cytokines, chemokines, and transcription factors (14). Similarly, stress-induced cellular pathways are upregulated in thawed PBMCs previously frozen in liquid nitrogen $(15,16)$. Most of these upregulated genes belong to the category of cellular immediate-early genes (IEGs) (17), also known as primary- or transient-response genes. IEGs are characterized by a rapid, transient increase in gene transcription upon encounter with mitogens or external stress; this transcriptional response is unaltered by protein synthesis inhibitors (17). Mitogen-activated protein kinases (MAPKs) are the first sensors of mitogens and extracellular stress, and IEG transcription is MAPK dependent (18). Since the burst of HTLV-1 plus-strand expression in fresh PBMCs in vitro shows similar expression kinetics, we hypothesized that the integrated HTLV-1 provirus would behave like a cellular IEG.

Most work to date on the regulation of HTLV-1 proviral expression has been performed in transformed cell lines with recombinant plasmid-based expression systems or in primary infected CD4 ${ }^{+} \mathrm{T}$ cells incubated for $\geq 1$ day. To test the hypothesis that the HTLV-1 provirus exploits the intracellular signaling activation pathways employed by cellular IEGs, we used freshly obtained whole blood from HTLV-1-infected individuals and processed it within minutes of venepuncture. We quantified the kinetics of expression of the HTLV-1 plus-strand and classical IEGs and identified critical factors that activate or repress HTLV-1 transcription.

\section{Results}

Immediate-early HTLV-1 reactivation is p38-MAPK dependent and is not hindered by protein synthesis inhibition. The p38-MAPK pathway has previously been reported to influence HTLV-1 expression in both patient-derived cell lines and primary PBMCs $(19,20)$. However, in these studies HTLV-1 Gag expression was quantified after incubation for 1-5 days, in the presence or absence of p38-MAPK inhibitors. To investigate whether the p38 pathway plays a role in the apparently spontaneous burst of tax expression observed immediately after ex vivo culture of blood from HTLV-1-infected individuals, PBMCs collected into EDTA were separated within 15 minutes from fresh venous blood and cultured for 2 hours in the presence of either a p38-MAPK inhibitor (SB203580 or BIRB796) or an ERK-MAPK inhibitor (PD184352 or U0126) (21). The cells were then assayed for HTLV-1 transcription by quantitative PCR (qPCR) (Figure 1) (22). The broad-spectrum 2-oxoglutarate oxygenase inhibitor and HIF stabilizer dimethyloxalylglycine (DMOG) acted as the positive control for inhibition of HTLV-1 transcription, as reported previously $(23,24)$. The MAPK-dependent immediate-early gene $c$-fos was used as a positive control for a drug-mediated response (25). HTLV-1 tax transcription was significantly inhibited by the p38-selective inhibitors but not by the ERK-MAPK inhibitors (Figure 1, tax). As expected, mRNA levels of the IEG c-fos were inhibited by both the p38- and ERK-MAPK inhibitors because c-fos transcription is known to be regulated by both these MAPK pathways (Figure 1, c-fos) (18).

A key characteristic of IEGs is that their transcription is apparently not dependent on de novo protein synthesis (17). In agreement with this, treatment with the protein synthesis inhibitor anisomycin (26) did not inhibit immediate-early HTLV-1 transcription. In contrast, by virtue of its action as a MAPK inducer, anisomycin strongly potentiated HTLV-1 reactivation in freshly isolated PBMCs, similar to its effect on IEG $c$-fos transcription (26) (Figure 1).

PRC1 signature at the HTLV-1 provirus. IEGs are frequently regulated by polycomb repressive complexes (PRCs) (18). PRC1 represses transcription through monoubiquitylation of Lys119 in histone H2A (H2AK119ub1); PRC2 does so via a mechanism involving trimethylation of Lys27 on histone H3. Most IEG promoters are classified as bivalent because they contain both the transcriptionally activating epigenetic mark $\mathrm{H} 3 \mathrm{~K} 4 \mathrm{me} 3$ and the inhibitory mark H3K27me3. IEG activation is associated with an increase in $\mathrm{H} 3 \mathrm{~K} 4 \mathrm{me} 3$ and a reduction in H3K27me3 levels (18). By contrast, we had previously shown that HTLV-1 

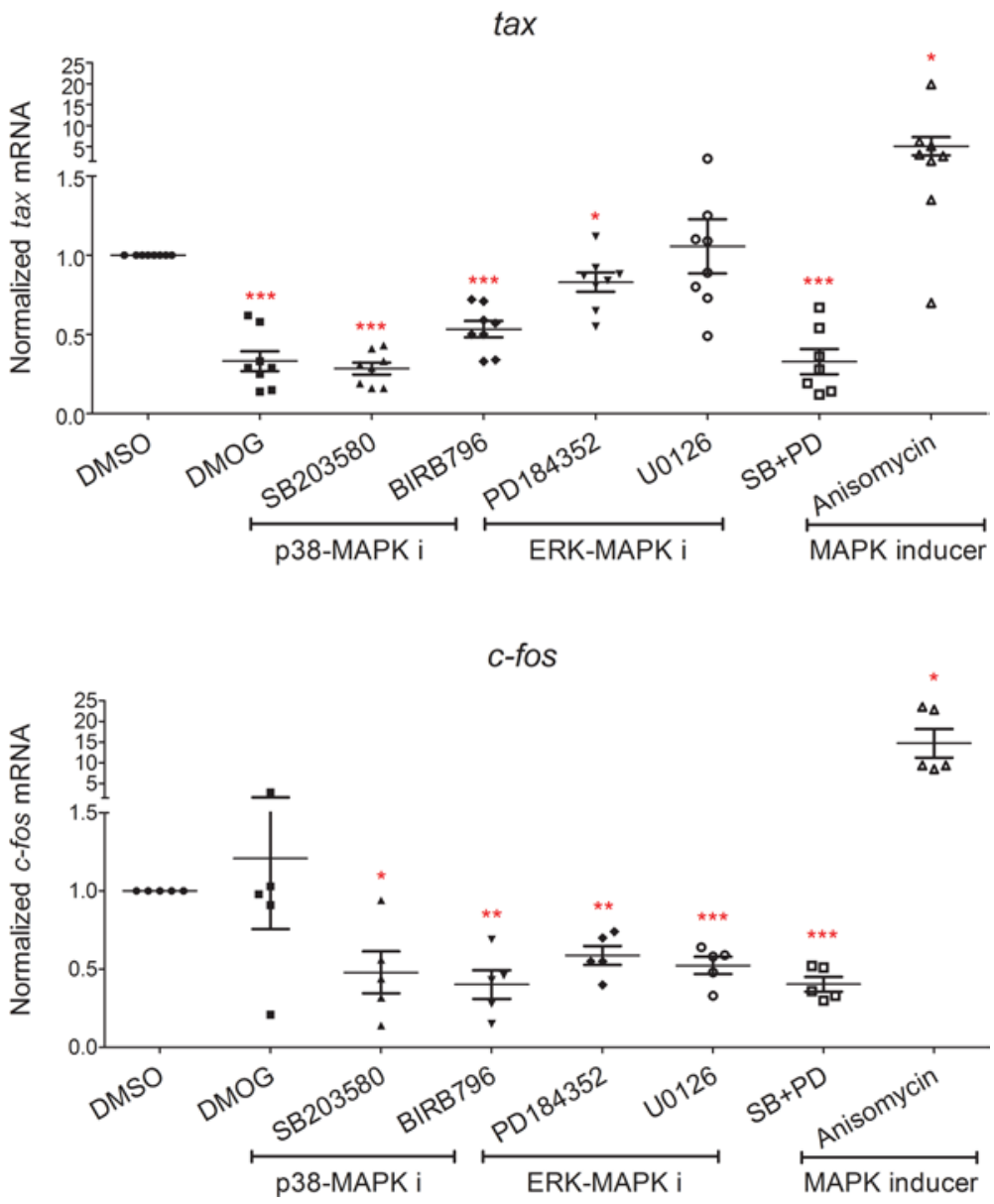

Figure 1. HTLV-1 plus-strand transcription is p38-MAPK dependent. PBMCs were isolated from freshly obtained venepuncture samples from HTLV-1-infected individuals. Isolated cells were cultured for 2 hours (hr) in the presence of either a control (DMSO), p38-MAPK inhibitors (10 $\mu \mathrm{M}$ SB203580; $5 \mu \mathrm{M}$ BIRB796), ERK-MAPK inhibitors (10 $\mu \mathrm{M}$ PD184352; $10 \mu \mathrm{M}$ U0126), or the cellular protein synthesis inhibitor and MAPK-inducer, anisomycin $(5 \mu \mathrm{M})$. DMOG was employed as the positive control for inhibition of plusstrand HTLV-1 transcription. RNA was isolated and subjected to $\mathrm{QPCR}$ with primers specific for tax mRNA (plus-strand) or c-fos mRNA (positive control). Data represent \pm SEM. Statistical significance was calculated using the 2-tailed ratio $t$ test (*** $^{*}<0.0005$; ${ }^{* *} P<0.005$; $\left.{ }^{*} P<0.05\right) . n=8$ for tax; $n=$ 5 for $c$-fos.

activation is associated with an increase in $\mathrm{H} 3 \mathrm{~K} 4 \mathrm{me} 3$ levels, but there was no associated reduction in H3K27me3 levels (23). To reconfirm and investigate this result, we used ChIP to assay the levels of the H3K27-specific histone methyltransferase EZH2 - the key enzyme in PRC2 - at the HTLV-1 provirus in cryopreserved HTLV-1-infected PBMCs, which were fixed either immediately or after overnight culture (Figure 2A). There was in fact a small but statistically significant increase in EZH2 at 17 hours when compared with T0 at the 5'-LTR junction (Figure 2A), implying that PRC2 does not inhibit the spontaneous burst of HTLV-1 plus-strand expression. We therefore assayed the characteristic signature of PRC1, which is H2AK119ub1, at the HTLV-1 provirus. We found that H2AK119ub1 levels were maximum at T0, but there was a rapid and significant reduction in the levels as early as 2 hours in the HTLV-1 provirus (Figure $2 \mathrm{~B}$ ). Thus, immediate-early proviral reactivation is associated with an increase in $\mathrm{H} 3 \mathrm{~K} 4 \mathrm{me} 3$ and a reduction in $\mathrm{H} 2 \mathrm{AK} 119 \mathrm{ub} 1$. The presence of both transcriptionally activating and repressive epigenetic modifications enables bivalent promoters to silence gene expression while maintaining the potential to rapidly reactivate transcription in response to specific environmental cues. The classical bivalent promoters are enriched in the inhibitory mark H3K27me3 and the activating mark H3K4me3 (27). However, the HTLV-1 5'-LTR promoter is atypical, with enrichment of the inhibitory mark H2AK119ub1 and the activatory mark H3K4me3. These observations suggest that HTLV-1 plus-strand transcription is governed by a PRC1-dependent, bivalent promoter.

Effects of pharmacological modulation of H2AK119ub1 levels on the HTLV-1 provirus. If HTLV-1 plus-strand expression is indeed inhibited by H2AK119ub1, then preventing the removal of this histone mark should block the spontaneous burst of plus-strand transcription that is observed after short-term in vitro culture. We used a broad-spectrum cysteine deubiquitinase (DUB) inhibitor (PR619) (28) and an inhibitor of H2A ubiquitylation (PRT4165) (29) to study their effects on HTLV-1 transcription. We have previously shown that limiting the concentration of glucose in culture medium impairs HTLV-1 plus-strand transcription (23). We therefore compared the effects on proviral transcription and epigenetic signatures of modulating the level of H2AK119ub1 either alone or in combination with varying the concentration of glucose. Cryopreserved 

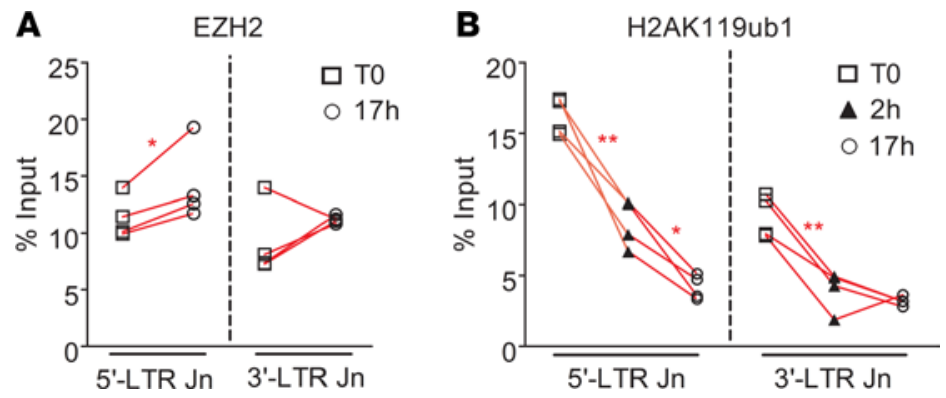

Figure 2. PRC1 signature at the HTLV-1 provirus. Cryopreserved HTLV-1-infected PBMCs were subjected to ChIP and the precipitate amplified by qPCR with primers specific respectively for the 5'-LTR and 3'-LTR junctions of the HTLV-1 provirus. (A) ChIP-qPCR using antibody against EZH2 (PRC2 enzyme) after $0 \mathrm{hr}$ (TO) and $17 \mathrm{hr}$ (17h) of culture. (B) ChIP-qPCR with antibody against H2AK119ub1 (PRC1 epigenetic mark) after $0 \mathrm{hr}, 2 \mathrm{hr}$, and $17 \mathrm{hr}$ of culture. Enrichment is expressed as percent input DNA in the ChIP. Statistical significance was calculated using the 2-tailed Student's $t$ test $\left({ }^{*} P<0.05 ;{ }^{* *} P<0.005\right) . n=4$ for both $\mathbf{A}$ and $\mathbf{B}$.

PBMCs from HTLV-1-infected individuals were cultured for 2 hours in the presence of medium with either (a) physiological glucose concentration (5.5 mM); (b) PR619; (c) no glucose + 2-deoxy-D-glucose (2-DG; glycolysis inhibitor) (30); or (d) no glucose + 2-DG + PRT4165 (Figure 3). We quantified transcriptional changes at the HTLV-1 provirus plus- and minus-strands by qPCR and used ChIP to quantify epigenetic changes at the HTLV-1 provirus; as the positive control, we used the promoter of the cellular ATF3 gene (a PRC1-dependent IEG) and its corresponding mRNA for qPCR $(31,32)$. Treatment with the DUB inhibitor PR619 resulted in a significant reduction in tax transcription (Figure 3A, tax), accompanied by a significant increase in H2AK119ub1 levels at the 5'-LTR and 3'-LTR junctions (Figure 3B, H2AK119ub1). The steep fall in H2AK119ub1 upon activation of the HTLV-1 provirus was not limited to the 5'- and 3'-LTR junctions but was also observed in other regions of the HTLV-1 provirus tested (data not shown). It has been shown before that the catalytic domains of H3K4-methyltransferases and H3K36-methyltransferases are inhibited by the presence of nucleosomes containing H2AK119ub1 (33-35). Thus, proviral reactivation is accompanied by a reduction in H2AK119ub1 and an associated increase in H3K4me3 and H3K36me3 across the provirus $(3,23)$. When HTLV-1-infected PBMCs were cultured for 2 hours in the presence of the cysteine DUB inhibitor PR619, there was no significant change in the levels of the activatory histone marks H3K4me3 or H3K9/14Ac, even though plus-strand transcription was strongly inhibited (Figure 3, A and B). Thus, partially reversing immediate-early changes in H2AK119ub1 by treatment with PR619 had no impact on H3K4me3 or H3K9/14Ac levels at the HTLV-1 5'-LTR junction. While we have shown previously that limiting cellular glucose levels inhibited HTLV-1 plus-strand transcription upon overnight culture, glucose removal had no effect on HTLV-1 transcription within 2 hours $(3,23)$ (Figure 3A, tax). Removing glucose from the medium also had no impact on H2AK119ub1 levels at the HTLV-1 5'-LTR junction (Figure 3B, H2AK119ub1). Surprisingly, addition of the PRC1 inhibitor PRT4165 to the medium without glucose resulted in a significant reduction in tax transcription levels (Figure 3A, tax). Because this reduction was accompanied by minimal changes in H2AK119ub1 levels, we conclude that it is likely to be a nonspecific or secondary effect of this reactive electrophilic alkylating agent (Figure 3B, H2AK119ub1). To investigate whether this effect was specific to PRT4165 and not caused by the absence of glucose, we cultured HTLV1-infected primary PBMCs for 2 hours in the presence of PRT4165 in medium with physiological glucose concentration $(5.5 \mathrm{mM})$. Even in medium with a physiological glucose concentration, PRT4165 significantly inhibited plus-strand transcription, suggesting that the observed effect was indeed inhibitor specific and not a function of glucose concentration (Supplemental Figure 1A; supplemental material available online with this article; https://doi.org/10.1172/jci.insight.123196DS1). The inhibitory effect of PRT4165 on tax transcription was, however, overcome after culture in the presence of the drug for 17 hours (Supplemental Figure 1B, tax). In contrast, the inhibitory effect of PR619 on HTLV-1 plus-strand transcription became even stronger upon overnight culture (Supplemental Figure 1B, tax).

The cellular ATF3 gene is a bona fide PRC1-dependent IEG (31). However, unlike HTLV-1, it is known that DUB-mediated repression inhibits ATF3 mRNA transcription (36). As expected, the DUB inhibitor PR619 relieved this repression and significantly increased ATF3 mRNA transcription. This increase in 

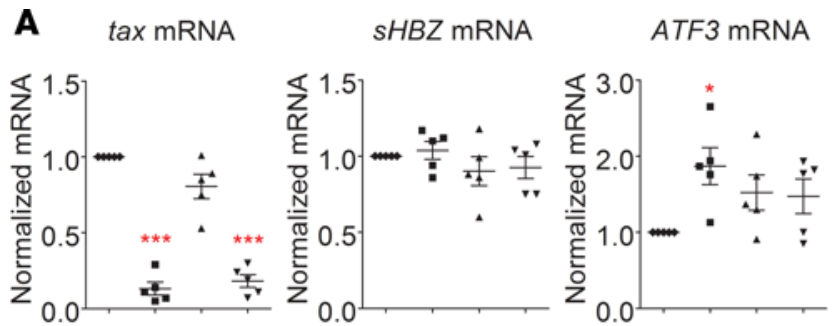

Control

- PR619

$\Delta$ No Glucose+ 2-DG

$\nabla$ No Glucose+

2-DG+ PRT4165
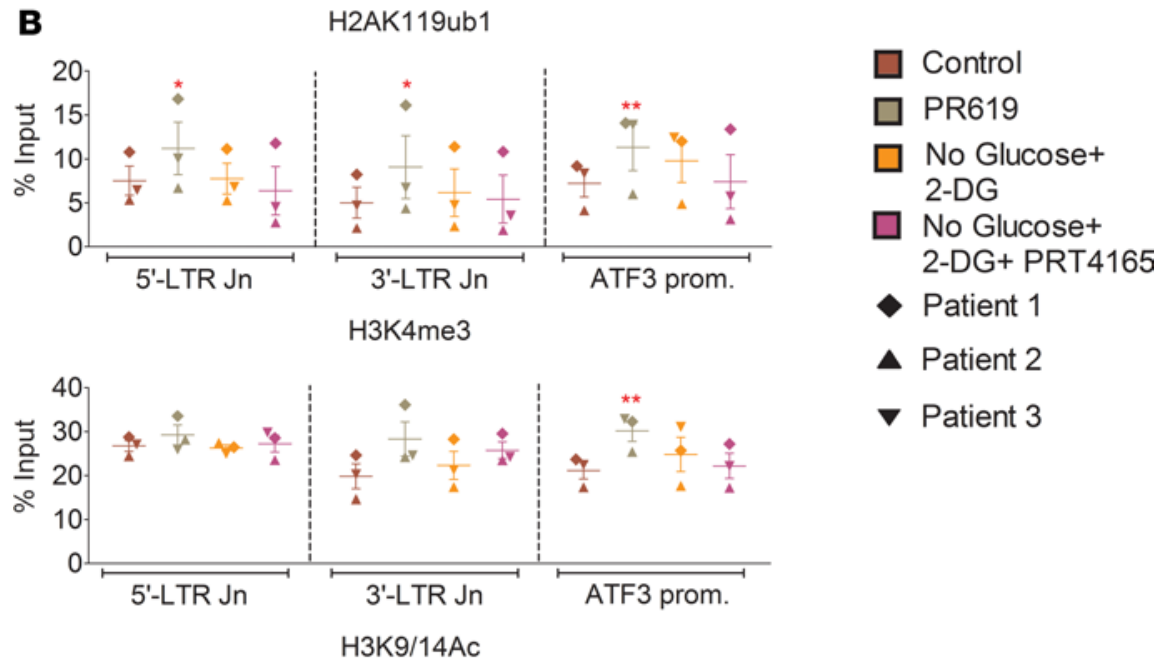

$\Delta$ Patient 2

v Patient 3

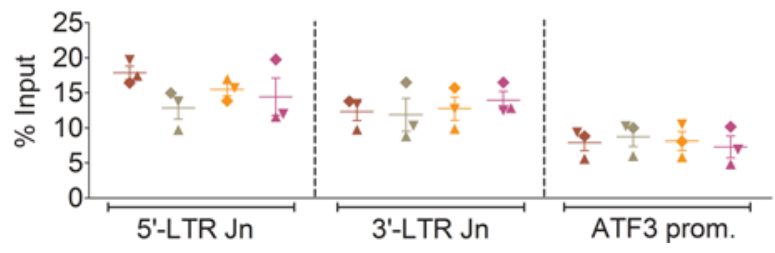

Figure 3. Immediate-early transcriptional and epigenetic effects of glucose and PRC1 at the HTLV-1 provirus. Cryopreserved PBMCs isolated from HTLV-1-infected individuals were cultured for $2 \mathrm{hr}$ in the presence of either control (DMSO); deubiquitinase inhibitor (PR619); glucose-free medium with 2-deoxyglucose (no glucose+2-DC); or no glucose+2-DG + PRT4165 (an inhibitor of ubiquitylation of H2AK119. (A) Cells were harvested for RNA and subjected to qPCR with primers specific for tax mRNA (plus-strand), sHBZ mRNA (minus-strand), and ATF3mRNA (positive control). Data represent \pm SEM. (B) Cells were fixed after culture for $2 \mathrm{hr}$ and subjected to ChIP-qPCR, using antibodies directed against H2AK119ub1, H3K4me3, H3K9/14Ac and IgG, and primers specific respectively for the $5^{\prime}$-LTR and 3'-LTR junctions of the HTLV-1 provirus. The promoter of the host gene ATF3 was used as the positive control for ChIP. Enrichment is expressed as percentage of input DNA. Data represent \pm SEM. Statistical significance was calculated using the 2-tailed ratio $t$ test $\left({ }^{* *} P<0.0005 ;{ }^{* *} P<0.005 ;{ }^{*} P<0.05\right) . n=5$ for $\mathbf{A}$ and $n=3$ for $\mathbf{B}$.

ATF3 transcription was associated with an increase in $\mathrm{H} 2 \mathrm{AK} 119 \mathrm{ub} 1$ and $\mathrm{H} 3 \mathrm{~K} 4 \mathrm{me} 3$ at the $A T F 3$ gene promoter (Figure 3, A and B).

Neither PR619 nor PRT4165 altered sHBZ transcription at 2 hours, but each drug significantly induced $s H B Z$ transcription after 17 hours (Figure 3A and Supplemental Figure 1, A and B). There was a significant increase in H2AK119ub1 levels at both the 5'- and 3'-LTRs after 2 hours of PR619 treatment. However, the transcriptional changes were observed only in the plus-strand. These results show that H2AK119ub1-mediated repression is HTLV-1 plus-strand specific. Both 2 hours and 17 hours after PR619 treatment, there was a consistent increase in H3K4me3 levels in all the samples tested at the 3'-LTR junction (Figure 3B and Supplemental Figure 1C, H3K4me3). The increase in H3K4me3 at the 3'-LTR junction, associated with a corresponding increase in $s H B Z$ transcription in response to the DUB inhibitor PR619, points toward an important but previously unrecognized role played by ubiquitylation in the regulation of the HTLV-1 3'-LTR promoter.

Distinct spatiotemporal epigenetic programs govern HTLV-1 reactivation from latency. The results presented above identify 3 pathways, which, when perturbed, alter the burst of HTLV-1 plus-strand transcription. Inhibition of either p38-MAPK activity or deubiquitylation of H2AK119ub1 inhibits immediate-early 
A
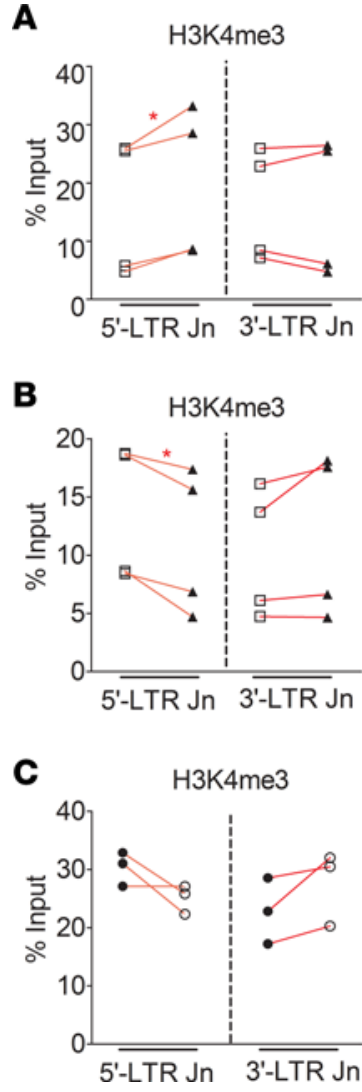
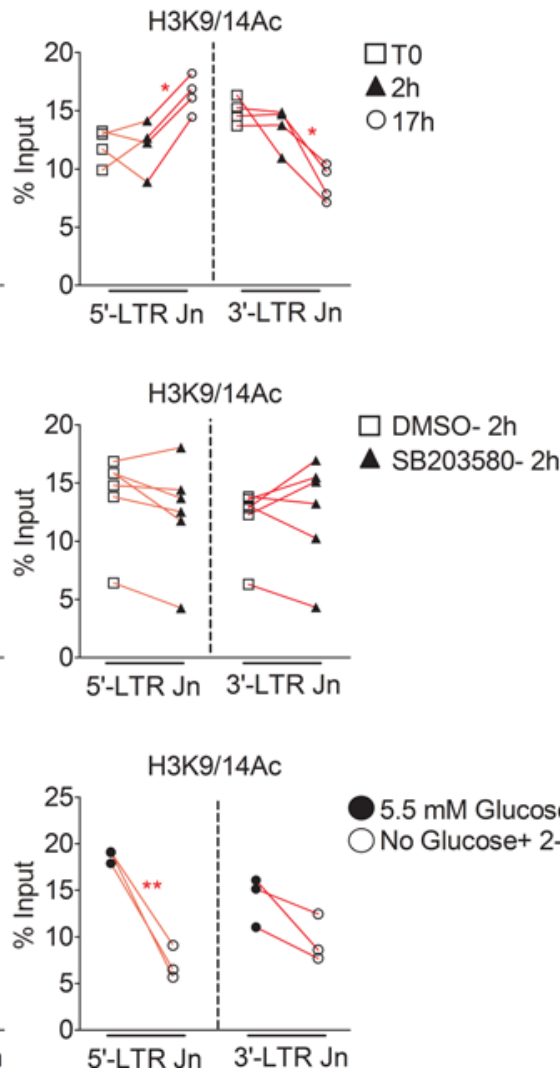

Figure 4. Distinct epigenetic signatures of p38-MAPK and glucose deprivation at the HTLV-1 provirus. Cryopreserved HTLV-1-infected PBMCs were fixed either (A) immediately (TO) or after $2 \mathrm{hr}$ (2h) or $17 \mathrm{hr}$ (17h) of culture; (B) after $2 \mathrm{hr}$ of culture in the presence of a control (DMSO) or a p38-MAPK inhibitor (SB203580); or (C) after $17 \mathrm{hr}$ of culture in 5.5 $\mathrm{mM}$ glucose-containing medium ( $5.5 \mathrm{mM}$ glucose-17h) or in glucose-free medium containing the glycolysis inhibitor 2-deoxy-D-glucose (no glucose + 2-DG-17h). Fixed cells were subjected to ChIP-qPCR, using antibodies directed against H3K4me3, H3K9/14Ac or IgG, and primers specific respectively for the 5'-LTR and 3'-LTR junctions of the HTLV-1 provirus. Enrichment is expressed as percentage of input DNA. Statistical significance was calculated using the 2-tailed Student's $t$ test ( $\left.{ }^{* *} P<0.005,{ }^{*} P<0.05\right) . n=4$ for $\mathbf{A}$ and $\mathbf{B}(\mathrm{H} 3 \mathrm{~K} 4 \mathrm{me} 3) ; n=6$ for $\mathbf{B}(\mathrm{H} 3 \mathrm{~K} 9 / 14 \mathrm{Ac})$; and $n=3$ for $\mathbf{C}$

HTLV-1 transactivation (2 hours) (Figures 1-3). By contrast, limiting the concentration of glucose does not alter immediate-early HTLV-1 reactivation (Figure 3A, tax) but strongly inhibits plus-strand transcription after prolonged culture (17 hours) (23). We wanted to test the hypothesis that these 3 pathways regulate HTLV-1 transcription by acting in concert on epigenetic modifications at the HTLV-1 provirus.

Cryopreserved HTLV-1-infected primary PBMCs were fixed either immediately or after culture for 2 hours or 17 hours. ChIP-qPCR was carried out to assay H3K4me3 and H3K9/14Ac at the 5'-LTR junction and $3^{\prime}$-LTR junction (Figure 4A). Within 2 hours of proviral reactivation, there was a rapid and significant increase in H3K4me3 at the 5'-LTR junction when compared with the 3'-LTR junction (Figure 4A, $\mathrm{H} 3 \mathrm{~K} 4 \mathrm{me} 3)$. In contrast, the level of $\mathrm{H} 3 \mathrm{~K} 9 / 14 \mathrm{Ac}$ was unchanged at this early time point. Instead, there was a significant increase in H3K9/14Ac levels at the HTLV-1 5'-LTR junction after 17 hours of culture (Figure $4 \mathrm{~A}, \mathrm{H} 3 \mathrm{~K} 9 / 14 \mathrm{Ac}$ ). Furthermore, this late increase in H3K9/14Ac at the $5^{\prime}$-LTR junction was associated with a significant reduction in H3K9/14Ac at the $3^{\prime}$-LTR junction from 2 hours to 17 hours. These results suggest that the $5^{\prime}$-LTR and 3'-LTR promoters are differentially regulated by histone deacetylases (HDACs) and histone acetyltransferases (HATs) (Figure 4A, H3K9/14Ac). These enzymes have also been implicated in the regulation of IEG expression $(37,38)$.

Consistent with the notion of differential regulation of the 2 respective HTLV-1 promoters, treatment with the p38-MAPK inhibitor SB203580 for 2 hours resulted in a significant reduction in H3K4me3 at the $5^{\prime}$-LTR junction when compared with DMSO-treated cells, suggesting that the p38 pathway regulates H3K4me3 levels at the HTLV-1 5'-LTR (Figure 4B). There was no corresponding change in H3K9/14Ac or 
A
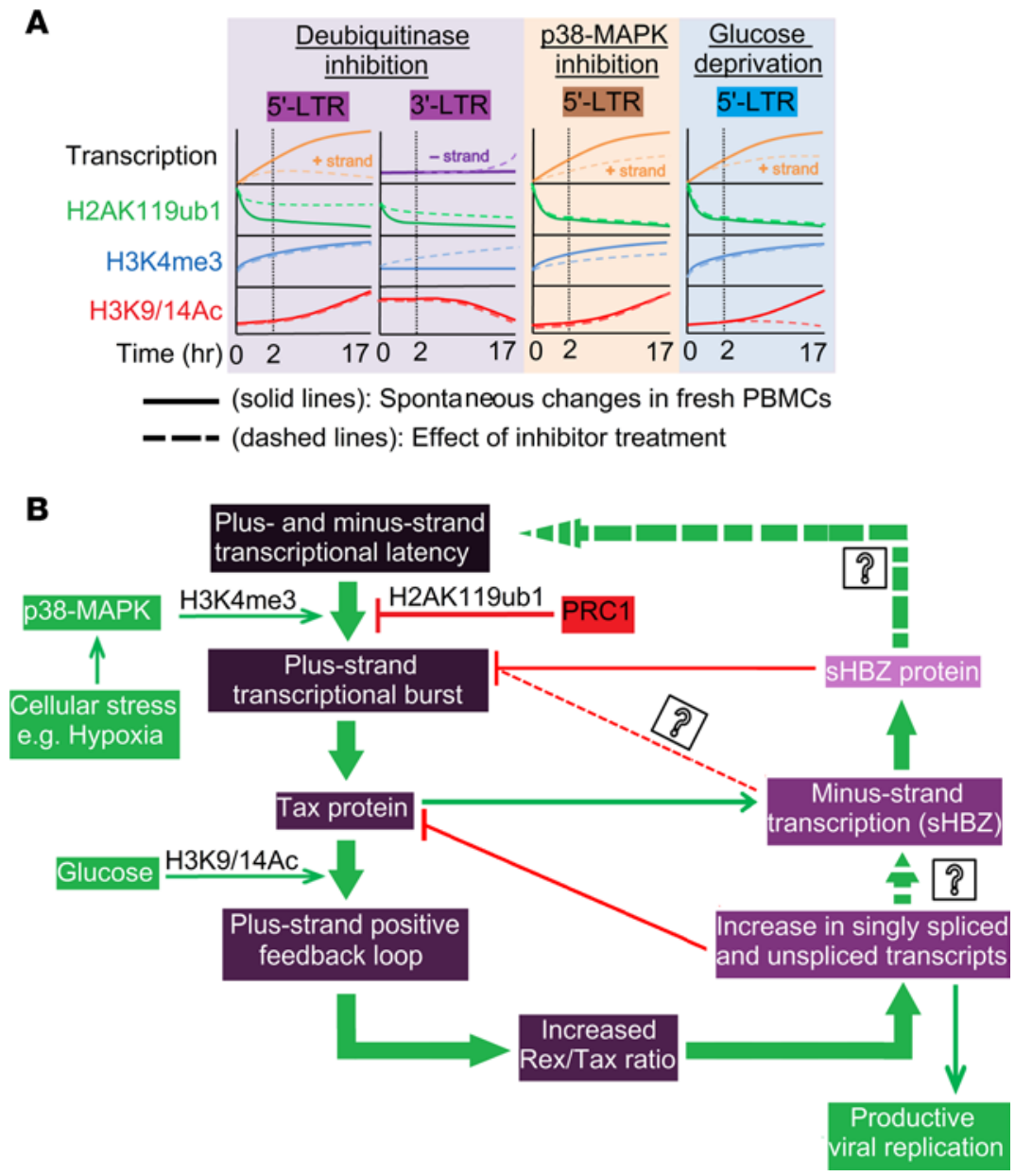

Figure 5. Summary of results and model of regulation of HTLV-1 latency and reactivation. (A) Schematic summary of transcriptional and epigenetic changes observed in this study upon spontaneous proviral reactivation in primary HTLV-1-infected PBMCs during culture and after inhibition of deubiquitinases, p38-MAPK, or glucose metabolism. (B) Model of HTLV-1 latency and reactivation. The evidence reported here suggests that cellular stress (e.g., physiological hypoxia; ref. 23) is an important trigger of HTLV-1 proviral reactivation from latency and that monoubiquitylated $\mathrm{H} 2 \mathrm{~A}$ (H2AK119ub1), deposited by polycomb repressive complex 1 (PRC1), is a major barrier to this reactivation. Green arrows indicate induction; red lines indicate inhibition. Dashed lines denote hypothetical actions.

H2AK119ub1 levels upon p38 inhibitor treatment (Figure 4B and Supplemental Figure 2A).

Next, cryopreserved HTLV-1-infected PBMCs were cultured either in the presence of RPMI with either a physiological glucose concentration (5.5 mM glucose) or RPMI with no glucose and added 2-deoxy-Dglucose (glycolysis inhibitor). Since culture for 2 hours in glucose-free medium had no impact on HTLV-1 plus-strand transcription, cells were cultured for 17 hours in the conditions stated above. Inhibition of glucose metabolism had minimal effects on $\mathrm{H} 3 \mathrm{~K} 4 \mathrm{me} 3$ levels (Figure 4C, H3K4me3). However, there was a strong and significant reduction in $\mathrm{H} 3 \mathrm{~K} 9 / 14 \mathrm{Ac}$ at the 5 '-LTR junction in cells cultured in $0 \mathrm{mM}$ glucose + 2-DG, compared with cells cultured in physiological glucose concentration (Figure 4C, H3K9/14Ac). Cells cultured in glucose-free medium had very low H2AK119ub1 levels compared with cells incubated overnight in physiological glucose medium (Supplemental Figure 2B). However, since glucose has no impact on immediate-early H2AK119ub1 levels, this delayed-onset reduction in H2AK119ub1 observed after overnight culture in glucose-free medium might be due to secondary effects of the low glucose concentration on cellular ubiquitylation pathways (Figure 3B, H2AK119ub1; Supplemental Figure 2B).

To summarize, H3K4me3 is the most dynamic activating mark associated with immediate-early proviral reactivation from latency. The increase in the $\mathrm{H} 3 \mathrm{~K} 9 / 14 \mathrm{Ac}$ mark follows the increase in $\mathrm{H} 3 \mathrm{~K} 4 \mathrm{me} 3$ and reaches a peak some 17 hours after the onset of reactivation at the $5^{\prime}$-LTR junction. p38-MAPK inhibition is associated with a reduction in immediate-early H3K4me3 levels at the $5^{\prime}$-LTR. Limiting glucose in the 
medium results in a delayed but strong reduction of $\mathrm{H} 3 \mathrm{~K} 9 / 14 \mathrm{Ac}$ levels at the 5'-LTR junction. Finally, treatment with the DUB inhibitor PR619 does not alter either of these marks but independently reduces HTLV-1 plus-strand transcription and blocks the deubiquitylation of H2AK119ub1 (Figure 3B). The results show that these 3 transcriptional and epigenetic programs act independently to govern HTLV-1 transactivation from latency.

\section{Discussion}

Each mammalian gene is marked by a characteristic pattern of epigenetic modifications in its promoter regions and gene body. These epigenetic modifications are frequently associated with transcriptional activity, but in many cases, the causal relationship between the epigenetic modification and transcription is unclear. We sought to investigate the pathways that influence reactivation of HTLV-1 in fresh ex vivo PBMCs by identifying the epigenetic signatures in the provirus associated with HTLV-1 gene expression, regardless of the integration site.

The observation of sudden spontaneous proviral plus-strand transcription bursts in ex vivo cultured PBMCs obtained from HTLV-1-infected individuals suggested that HTLV-1 might be exploiting intracellular signaling pathways employed by IEGs, which are activated when blood is cultured ex vivo $(15,16)$. The observations reported here suggest that HTLV-1 is indeed regulated like an IEG in 3 key respects. First, spontaneous immediate-early plus-strand transcriptional bursting was independent of cellular protein synthesis (Figure 1). Second, this primary response was strongly inhibited by p38-specific MAPK inhibitors and potentiated by the MAPK inducer anisomycin (Figure 1). Third, certain epigenetic characteristics of the HTLV-1 provirus strongly resemble those of IEGs. Most cellular IEG promoters exhibit a balance between the transcriptionally activating histone epigenetic mark $\mathrm{H} 3 \mathrm{~K} 4 \mathrm{me} 3$ and the repressive $\mathrm{H} 3 \mathrm{~K} 27 \mathrm{me} 3$ mark (18) and are therefore termed bivalent promoters. IEG activation is associated with an increase in $\mathrm{H} 3 \mathrm{~K} 4 \mathrm{me} 3$ and a reduction in $\mathrm{H} 3 \mathrm{~K} 27 \mathrm{me}$. With HTLV-1, we have previously shown that the provirus is enriched in both the $\mathrm{H} 3 \mathrm{~K} 4 \mathrm{me} 3$ and $\mathrm{H} 3 \mathrm{~K} 27 \mathrm{me} 3$ histone modifications (23). We show here that proviral reactivation is associated with an increase in $\mathrm{H} 3 \mathrm{~K} 4 \mathrm{me} 3$ but not with a reduction in $\mathrm{H} 3 \mathrm{~K} 27 \mathrm{me} 3$ levels (23). Poised promoters are either regulated by $\mathrm{H} 3 \mathrm{~K} 27 \mathrm{me} 3$, the histone signature of PRC2, or H2AK119ub1, the histone signature of PRC1 (39). H2A ubiquitylation at lysine 119 inhibits RNA polymerase II elongation at bivalent genes $(40,41)$. We found that H2AK119ub1 was highly enriched in frozen PBMCs immediately after thawing, but after subsequent culture for as little as 2 hours, there was a strong and significant drop in the level of this mark, coincident with proviral reactivation (Figure 2). Inhibition of the spontaneous deubiquitylation of H2A by a broad-spectrum DUB inhibitor (PR619) was associated with a strong and statistically significant reduction in immediate-early HTLV-1 plus-strand transcription (Figure 3). It has been reported that PRC2 plays an important role in the establishment and maintenance of epigenetic silencing in HIV-1 $(42,43)$. In contrast, our results indicate that PRC1, but not PRC2, plays an important part in regulating the proviral latency of HTLV-1.

Cellular IEGs are often the first genes that respond to cellular stress (17). Thus, any extracellular stress that activates p38-MAPK activity such as hypoxia or changes in temperature or osmolarity might reactivate latent HTLV-1 in vivo. Our results suggest that integrated HTLV-1 hijacks IEG mechanisms to sense a change in the cellular environment and consequently initiate productive replication. Since proviral transcription is frequently undetectable in freshly isolated infected CD4 $4^{+} \mathrm{T}$ cells, it is possible that infectious spread is more likely to occur in certain niches in the host or when the cell experiences stresses - for example, during transfer to a new host. We previously showed that physiological hypoxia - the low oxygen tension present in BM, lymph nodes, and other end organs - enhances plus-strand HTLV-1 gene transcription (23). Recently, it was also reported that tax is highly expressed in BM when compared with other tissues in Japanese macaques infected with the closely related virus simian T-lymphotropic virus type 1 (STLV-1) (44).

The evidence presented here and in earlier studies by ourselves and others shows that interventions in 3 pathways can inhibit HTLV-1 plus-strand reactivation from latency, namely inhibition of p38-MAP kinases, preventing spontaneous H2A deubiquitylation and limiting glucose metabolism. To identify whether these pathways act on the HTLV-1 provirus independently or in concert, we studied epigenetic signatures associated with each respective pathway. $\mathrm{H} 3 \mathrm{~K} 4 \mathrm{me} 3$ was the most dynamic mark, increasing strongly during immediate-early proviral reactivation (Figure $4 \mathrm{~A}$ and Figure $5 \mathrm{~A}$ ). In comparison, H3K9/14Ac levels remained constant during immediate-early HTLV-1 reactivation but subsequently rose during the positive-feedback phase of proviral transcription (Figure $4 \mathrm{~A}$ and Figure $5 \mathrm{~A}$ ). Consistent with the notion that the pathways 
act independently, p38-MAPK inhibition had no impact on H3K9/14Ac levels but significantly inhibited H3K4me3 levels in the immediate-early phase of HTLV-1 reactivation (Figure 4B and Figure 5A). By contrast, limiting glucose levels had no impact on the immediate-early HTLV-1 transcription but strongly inhibited plus-strand transcription after 17 hours, suggesting that glucose metabolism regulates the positive feedback phase of viral transcription (Figure 3A and Figure 5A) (23). Indeed, the strongest effect of limiting glucose metabolism was to reduce H3K9/14Ac levels at the HTLV-1 provirus (Figure 4C and Figure 5A). Thus, the effects of p38-MAPK inhibition and limiting glucose levels on HTLV-1 transcription are temporally distinct and have different epigenetic signatures. Similarly, pharmacological modulation of H2AK119ub1 levels alters immediate-early proviral reactivation but has no effect on $\mathrm{H} 3 \mathrm{~K} 4 \mathrm{me} 3$ or $\mathrm{H} 3 \mathrm{~K} 9 / 14 \mathrm{Ac}$ during the immediate-early phase (Figure 3). This observation suggests that H2AK119ub1 provides an independent barrier to HTLV-1 proviral reactivation.

In this study, PBMCs were taken from patients with HAM, with the exception of the results shown in Figure 1, in which cells from both asymptomatic HTLV-1 carriers and patients with HAM were employed. While HTLV-1 expression can differ quantitatively between these clinical states (45), it is unlikely that the fundamental mechanisms of regulation of transcription differ qualitatively because the proviral sequences do not differ systematically between asymptomatic carriers (ACs) and patients with HAM (46).

The results presented here, and in previous work by ourselves (23) and others (47, 48), identify at least 4 distinct cellular pathways that exert opposing effects on HTLV-1 transcription from the plus- and minus- strands. First, the DUB inhibitor PR619, as reported in this study, inhibited plus-strand transcription while inducing minus-strand transcription. Second, DMOG, a structurally and functionally unrelated broad-spectrum 2-oxoglutarate oxygenase inhibitor and HIF stabilizer, had effects similar to those of PR619 on HTLV-1 plus- and minus-strand transcription (23). Third, the Wnt transcription factors TCF1 and LEF1 antagonized plus-strand transcription while enhancing minus-strand transcription (48). Fourth, the HDAC inhibitor valproate enhanced plus-strand transcription while inhibiting minus-strand transcription (47). These observations suggest that inhibition of the HTLV-1 plus-strand promotes minus-strand transcription and vice versa. It is known that perturbation of transcription on 1 DNA strand can interfere with transcription from the complementary strand $(49,50)$ - for example, by collision of convergent RNA polymerase II transcription complexes (51).

Yoshida et al. previously reported that HTLV-1 Tax activated the 3'-LTR promoter and induced sHBZ transcription (52). However, those experiments involved plasmid-based assays that could not quantify the 5 '-and 3'-LTR promoter activities in the context of their normal convergent orientation in the provirus. More recently, it was shown that the chromatin architectural protein CTCF binds the HTLV-1 provirus (22) and alters the structure and transcription of host chromatin through the formation of CTCF-mediated chromatin loops (53). The binding site of CTCF coincides with a sharp border in epigenetic modifications within the HTLV-1 provirus. It is yet unclear if CTCF, in addition to its effect on host chromatin and transcription, directly or indirectly regulates HTLV-1 latency and reactivation.

We summarize the present results and our interpretation in Figure 5. HTLV-1 plus-strand transcription is largely silent in the peripheral venous blood of infected individuals. Monoubiquitylated H2A (H2AK119ub1) is highly enriched at the latent 5'-LTR promoter and provides an epigenetic barrier to its reactivation. Encounter with stresses - for example, physiological hypoxia (23) - activates cellular p38-MAP kinases, increases H3K4me3 levels at the 5'-LTR promoter, and overcomes the H2AK119ub1 barrier, initiating immediate-early plus-strand transcription (Figure 5A). This initial phase of proviral reactivation leads to the production of fully spliced tax/rex HTLV mRNAs. Tax is more efficiently translated from the tax/rex mRNA due to its stronger Kozak consensus sequence compared with Rex (54). The immediate-early plus-strand reactivation is followed by the Tax protein-mediated positive feedback plus-strand transcriptional burst (12). Tax recruits CREB and the transcriptionally activating HATs CBP/p300 and P/CAF to the cAMP-response elements (CRE) within the 5'-LTR promoter, along with activation of the NF- $\mathrm{kB}$ and serum response factor (SRF) pathways $(55,56)$. This recruitment of HATs to the $5^{\prime}$-LTR promoter in the Tax-mediated positive feedback phase is associated with an increase in H3K9/14Ac at the $5^{\prime}$-LTR promoter (Figure 4A and Figure $5 \mathrm{~A}$ ). As more tax/rex transcripts are produced, Rex protein levels accumulate, resulting in the nuclear export of singly spliced and unspliced mRNAs and their translation to viral structural proteins, leading to productive viral replication (57). This increase in the export of singly spliced and unspliced mRNAs is associated with a reduction in the export of tax/rex mRNAs, resulting in a decrease in their protein levels in cells. In addition, the HTLV-1 accessory protein p30(II) binds to tax/rex mRNA and further prevents its export, 
leading to reduction in Tax protein levels (58). The reduction in Tax levels gradually diminishes plus-strand transcription. We postulate that this reduction in plus-strand transcription results in an increase in antisense $s H B Z$ transcription. It has been reported before that HBZ protein inhibits plus-strand transcription (59-62). sHBZ mRNA may also inhibit plus-strand transcription (Figure 5B). Recently, Billman et al. showed at the single-cell level that HTLV-1 plus-strand mRNA transcription is enhanced in the absence of minus-strand transcripts, whereas high-level plus-strand transcription was accompanied by an increase in transcription from the minus-strand, generating a putative negative feedback inhibition of plus-strand expression $(3,12)$. However, the plus-strand mRNA probes employed for RNA-FISH in that study did not distinguish between early fully spliced transcripts and late singly spliced or unspliced transcripts. Billman et al. also found that, contrary to previous belief, not all HTLV-1-infected cells express $h b z$ mRNA at all times, suggesting that there is also a state of complete transcriptional latency of both plus- and minus-strands (Figure 5B).

The regulation of cellular gene expression involves time-dependent cross-talk between signaling events, metabolic status, epigenetic modifications, and the chromatin environment $(63,64)$. Our studies on the regulation of transcription of the same genetic element, the HTLV-1 provirus, in different genomic locations in naturally infected T cells reveals the importance of IEG-type mechanisms in regulating HTLV-1 transcription. We postulate that HTLV-1 has evolved to exploit immediate-early response mechanisms to regulate its latency in vivo, allowing the virus to minimize its exposure to host immune attack during chronic infection and to reactivate rapidly when a change in cellular microenvironment indicates an opportunity to spread to a new cell or a new individual. The 3 pathways investigated here each have a specific associated epigenetic signature at the integrated HTLV-1 provirus; the 3 pathways appear to function as independent checkpoints to viral expression and, therefore, allow the fine-tuning of transcriptional activity in response to multiple stimuli.

\section{Methods}

Primary $T$ cell isolation and culture. Venous blood samples from HTLV-1-infected individuals attending the clinic were collected into EDTA and separated using Histopaque, and the PBMCs were viably stored in liquid nitrogen. In case of freshly isolated samples, Histopaque separation and PBMC isolation was carried out within minutes of venepuncture, and the isolated PBMCs were used immediately for downstream processing and experiments. Cells were subjected to $\mathrm{CD} 8^{+} \mathrm{T}$ cell depletion using Dynabeads CD8 (Thermo Fisher Scientific) using manufacturer's instructions. Equal volumes of RPMI with L-glutamine without glucose (BE12-752F, Lonza) and RPMI with L-glutamine and $11 \mathrm{mM}$ glucose (BE12-702F) were mixed to make RPMI with L-glutamine and $5.5 \mathrm{mM}$ glucose; this medium was used for cell culture with $10 \%$ FBS (Thermo Fisher Scientific) in all experiments, unless otherwise stated.

Inhibitors. A comprehensive list of the compounds used is provided in Supplemental Table 2. A concentrated stock of each inhibitor was prepared in DMSO or water and was diluted to the desired concentration in culture medium.

$R N A$ extraction and $q P C R$. RNA extraction and qPCR was performed as described (23). Briefly, RNA was extracted from cultured PBMCs using the RNeasy Plus Mini kit (Qiagen). cDNA was synthesized from the extracted RNA using the Transcriptor First Strand cDNA Synthesis kit (Roche Diagnostics) by following the manufacturer's instructions. An additional control without reverse transcriptase was included for each cDNA sample synthesized. The kinetic PCR amplification was carried out using the Viia7 Real-time PCR system (Applied Biosystems) with gene-specific primers and Fast SYBR Green Master Mix. The LinRegPCR-Ct method (65) was used for relative quantification of target mRNA levels. LinRegPCR software was used to determine baselines, threshold, and mean efficiency $(\mathrm{E})$ of the reaction to calculate target mRNA quantity (R0), where R0 $=$ Threshold $/ \mathrm{E}^{\mathrm{Ct}}$. All values were normalized to their respective $18 \mathrm{~S}$ rRNA levels, which was the internal PCR control. The list of primers and probes used in this study is provided in Supplemental Table 3.

ChIP-qPCR. ChIP was performed as described (23). Cells were crosslinked with $1 \%(\mathrm{v} / \mathrm{v})$ aqueous formaldehyde for 10 minutes at room temperature. Nuclear lysates were sonicated using a Covaris S220 sonicator in a microTUBE (Covaris). Sonolab7.2 program parameters include peak incident power of 105 watts; duty factor of $10 \%$; cycles per burst of 200; treatment time of 480 seconds; and distilled water temperature of $4^{\circ} \mathrm{C}-6^{\circ} \mathrm{C}$. Sonicated lysates were subjected to IP using the following antibodies: ChIPAb + Trimethyl-Histone H3 Lys4 (MilliporeSigma, 17-614), anti-Acetyl-Histone H3 (Lys9/Lys14)(Cell Signaling Technology, 9677), Anti-Ubiquityl-Histone H2A (Lys119) (D27C4) XP rabbit mAb (Cell Signaling Technology, 8240), ChIPAb ${ }^{+}$EZH2 (clone AC22, MilliporeSigma, 17-662), ChIPAb + Trimethyl-Histone H3 Lys27 (MilliporeSigma, 17-622), and corresponding control IgG overnight at $4^{\circ} \mathrm{C}$ in the presence of 
MagnaChIP A+G magnetic beads (MilliporeSigma). A 10\% IP input sample was collected separately as a reference for relative quantification. The DNA enrichment in ChIP samples was quantified using region-specific primers respectively for the 5'-LTR junction and 3'-LTR junction of the HTLV-1 provirus and corresponding qPCR TaqMan probes (sequences described in Supplemental Table 3). The kinetic PCR amplification was carried out using the Viia7 Real-time PCR system (Applied Biosystems) with TaqMan Gene Expression Master Mix (Thermo Fisher Scientific). DNA enrichment was calculated as percentage of input $=\left(\mathrm{E}^{\Delta \mathrm{Ct}}\right) \times 10$, where $\Delta \mathrm{Ct}=\mathrm{Ct}($ input $)-\mathrm{Ct}($ sample $)$. LinRegPCR software was used to determine the mean efficiency of the reaction for each primer pair.

Statistics. Two-tailed Student's $t$ and ratio $t$ tests were employed for statistical analysis of data, as described in the figure legends.

Study approval. All donors attended the National Centre for Human Retrovirology (Imperial College Healthcare NHS Trust, St. Mary's Hospital, London, United Kingdom) and donated their blood to the Communicable Diseases Research Tissue Bank after giving written informed consent in accordance with the Declaration of Helsinki and with the approval of UK National Research Ethics Service (15/SC/0089). A list of patient samples tested has been provided in Supplemental Table 1. For most experiments, samples with a proviral load $\geq 3$ copies/100 PBMCs were used for ChIP and qPCR experiments. For all experiments in this study, except for the experiment depicted in Figure 1 where both ACs and HAM patient samples were analyzed as a proof of concept, only HAM patient samples were used.

\section{Author contributions}

$\mathrm{AK}$ and CRMB conceived and designed the experiments; AK performed the experiments; AK, CJS, RJK, and CRMB analyzed the data; CJS and RJK contributed reagents/materials/analysis tools; AK and CRMB wrote original draft; AK, CRMB, CJS, RJK, and GPT reviewed and edited manuscript; and GPT recruited patients.

\section{Acknowledgments}

We thank members of the CRMB, RJK, and CJS laboratories for helpful discussions. This work was supported by a project grant from the Medical Research Council, United Kingdom (MR/K019090/1), and a Senior Investigator Award from the Wellcome Trust, United Kingdom (WT100291MA), to CRMB and the Imperial National Institute for Health Research Biomedical Research Centre (https://imperialbrc.nihr.ac.uk/).

Address correspondence to: Charles R.M. Bangham, Division of Infectious Diseases, Faculty of Medicine, Imperial College London, Norfolk Place, London W2 1PG, United Kingdom. Phone: 44.0.20.7594.3730; Email: c.bangham@imperial.ac.uk.

1. Bangham CRM. Human T Cell Leukemia Virus Type 1: Persistence and Pathogenesis. Annu Rev Immunol. 2018;36:43-71.

2. Katsuya H, et al. Treatment and survival among 1594 patients with ATL. Blood. 2015;126(24):2570-2577.

3. Kulkarni A, Bangham CRM. HTLV-1: Regulating the Balance Between Proviral Latency and Reactivation. Front Microbiol. 2018;9:449.

4. Anderson MG, Dynan WS. Quantitative studies of the effect of HTLV-I Tax protein on CREB protein--DNA binding. Nucleic Acids Res. 1994;22(15):3194-3201.

5. Beimling P, Moelling K. Direct interaction of CREB protein with $21 \mathrm{bp}$ Tax-response elements of HTLV-ILTR. Oncogene. 1992;7(2):257-262.

6. Bangham CR, Cook LB, Melamed A. HTLV-1 clonality in adult T-cell leukaemia and non-malignant HTLV-1 infection. Semin Cancer Biol. 2014;26:89-98.

7. Hanon E, et al. Abundant tax protein expression in CD4+ T cells infected with human T-cell lymphotropic virus type I (HTLV-I) is prevented by cytotoxic T lymphocytes. Blood. 2000;95(4):1386-1392.

8. Minato S, Itoyama Y, Goto I, Yamamoto N. Expression of HTLV-I antigen in cultured peripheral blood mononuclear cells from patients with HTLV-I associated myelopathy. J Neurol Sci. 1988;87(2-3):233-244.

9. Tochikura T, Iwahashi M, Matsumoto T, Koyanagi Y, Hinuma Y, Yamamoto N. Effect of human serum anti-HTLV antibodies on viral antigen induction in vitro cultured peripheral lymphocytes from adult T-cell leukemia patients and healthy virus carriers. Int J Cancer. 1985;36(1):1-7.

10. Jacobson S, Shida H, McFarlin DE, Fauci AS, Koenig S. Circulating CD8+ cytotoxic T lymphocytes specific for HTLV-I pX in patients with HTLV-I associated neurological disease. Nature. 1990;348(6298):245-248.

11. Bangham CR. CTL quality and the control of human retroviral infections. Eur J Immunol. 2009;39(7):1700-1712.

12. Billman MR, Rueda D, Bangham CRM. Single-cell heterogeneity and cell-cycle-related viral gene bursts in the human leukaemia virus HTLV-1. Wellcome Open Res. 2017;2:87.

13. Mahgoub M, et al. Sporadic on/off switching of HTLV-1 Tax expression is crucial to maintain the whole population of 
virus-induced leukemic cells. Proc Natl Acad Sci USA. 2018;115(6):E1269-E1278.

14. Härtel C, Bein G, Müller-Steinhardt M, Klüter H. Ex vivo induction of cytokine mRNA expression in human blood samples. J Immunol Methods. 2001;249(1-2):63-71.

15. Yang J, et al. The effects of storage temperature on PBMC gene expression. BMC Immunol. 2016;17:6.

16. Tanner MA, Berk LS, Felten DL, Blidy AD, Bit SL, Ruff DW. Substantial changes in gene expression level due to the storage temperature and storage duration of human whole blood. Clin Lab Haematol. 2002;24(6):337-341.

17. Herschman HR. Primary response genes induced by growth factors and tumor promoters. Annu Rev Biochem. 1991;60:281-319.

18. Bahrami S, Drabløs F. Gene regulation in the immediate-early response process. Adv Biol Regul. 2016;62:37-49.

19. Fukushima N, Nishiura Y, Nakamura T, Yamada Y, Kohno S, Eguchi K. Involvement of p38 MAPK signaling pathway in IFN-gamma and HTLV-I expression in patients with HTLV-I-associated myelopathy/tropical spastic paraparesis. J Neuroimmunol. 2005;159(1-2):196-202.

20. Washiyama M, et al. IL-2 withdrawal induces HTLV-1 expression through p38 activation in ATL cell lines. FEBS Lett. 2007;581(27):5207-5212.

21. Burkhard K, Shapiro P. Use of inhibitors in the study of MAP kinases. Methods Mol Biol. 2010;661:107-122.

22. Satou Y, et al. The retrovirus HTLV-1 inserts an ectopic CTCF-binding site into the human genome. Proc Natl Acad Sci USA. 2016;113(11):3054-3059.

23. Kulkarni A, et al. Glucose Metabolism and Oxygen Availability Govern Reactivation of the Latent Human Retrovirus HTLV-1. Cell Chem Biol. 2017;24(11):1377-1387.e3.

24. Jaakkola P, et al. Targeting of HIF-alpha to the von Hippel-Lindau ubiquitylation complex by O2-regulated prolyl hydroxylation. Science. 2001;292(5516):468-472.

25. Renthal W, et al. Delta FosB mediates epigenetic desensitization of the c-fos gene after chronic amphetamine exposure. $J$ Neurosci. 2008;28(29):7344-7349.

26. Zinck R, Cahill MA, Kracht M, Sachsenmaier C, Hipskind RA, Nordheim A. Protein synthesis inhibitors reveal differential regulation of mitogen-activated protein kinase and stress-activated protein kinase pathways that converge on Elk-1. Mol Cell Biol. 1995;15(9):4930-4938.

27. Bernstein BE, et al. A bivalent chromatin structure marks key developmental genes in embryonic stem cells. Cell. 2006;125(2):315-326

28. Seiberlich V, Goldbaum O, Zhukareva V, Richter-Landsberg C. The small molecule inhibitor PR-619 of deubiquitinating enzymes affects the microtubule network and causes protein aggregate formation in neural cells: implications for neurodegenerative diseases. Biochim Biophys Acta. 2012;1823(11):2057-2068.

29. Ismail IH, McDonald D, Strickfaden H, Xu Z, Hendzel MJ. A small molecule inhibitor of polycomb repressive complex 1 inhibits ubiquitin signaling at DNA double-strand breaks. J Biol Chem. 2013;288(37):26944-26954.

30. Zhong D, et al. The glycolytic inhibitor 2-deoxyglucose activates multiple prosurvival pathways through IGF1R. J Biol Chem. 2009;284(35):23225-23233.

31. Prickaerts P, et al. MK3 controls Polycomb target gene expression via negative feedback on ERK. Epigenetics Chromatin. 2012;5(1):12.

32. Miyazaki K, et al. Differential usage of alternate promoters of the human stress response gene ATF3 in stress response and cancer cells. Nucleic Acids Res. 2009;37(5):1438-1451.

33. Yuan G, et al. Histone H2A ubiquitination inhibits the enzymatic activity of H3 lysine 36 methyltransferases. $J$ Biol Chem. 2013;288(43):30832-30842.

34. Endoh M, et al. Histone H2A mono-ubiquitination is a crucial step to mediate PRC1-dependent repression of developmental genes to maintain ES cell identity. PLoS Genet. 2012;8(7):e1002774.

35. Zhang T, Cooper S, Brockdorff N. The interplay of histone modifications - writers that read. EMBO Rep. 2015;16(11):1467-1481.

36. Dai F, et al. BAP1 inhibits the ER stress gene regulatory network and modulates metabolic stress response. Proc Natl Acad Sci USA. 2017;114(12):3192-3197.

37. Crump NT, Hazzalin CA, Bowers EM, Alani RM, Cole PA, Mahadevan LC. Dynamic acetylation of all lysine-4 trimethylated histone H3 is evolutionarily conserved and mediated by p300/CBP. Proc Natl Acad Sci USA. 2011;108(19):7814-7819.

38. Khan DH, Davie JR. HDAC inhibitors prevent the induction of the immediate-early gene FOSL1, but do not alter the nucleosome response. FEBS Lett. 2013;587(10):1510-1517.

39. Grossniklaus U, Paro R. Transcriptional silencing by polycomb-group proteins. Cold Spring Harb Perspect Biol. 2014;6(11):a019331.

40. Zhou W, et al. Histone H2A monoubiquitination represses transcription by inhibiting RNA polymerase II transcriptional elongation. Mol Cell. 2008;29(1):69-80.

41. Stock JK, et al. Ring1-mediated ubiquitination of H2A restrains poised RNA polymerase II at bivalent genes in mouse ES cells. Nat Cell Biol. 2007;9(12):1428-1435.

42. Friedman J, et al. Epigenetic silencing of HIV-1 by the histone H3 lysine 27 methyltransferase enhancer of Zeste 2. $J$ Virol. 2011;85(17):9078-9089.

43. Tripathy MK, McManamy ME, Burch BD, Archin NM, Margolis DM. H3K27 Demethylation at the Proviral Promoter Sensitizes Latent HIV to the Effects of Vorinostat in Ex Vivo Cultures of Resting CD4+ T Cells. J Virol. 2015;89(16):8392-8405.

44. Yasunaga J-i, et al. Hematopoietic Stem Cell Infected with HTLV-1 Functions As a Viral Reservoir In Vivo. Blood. 2016;128(22):1343.

45. Asquith B, et al. Quantification of the virus-host interaction in human T lymphotropic virus I infection. Retrovirology. 2005;2:75.

46. Daenke S, Nightingale S, Cruickshank JK, Bangham CR. Sequence variants of human T-cell lymphotropic virus type I from patients with tropical spastic paraparesis and adult T-cell leukemia do not distinguish neurological from leukemic isolates. $J$ Virol. 1990;64(3):1278-1282.

47. Belrose G, et al. Effects of valproate on Tax and HBZ expression in HTLV-1 and HAM/TSP T lymphocytes. Blood. 2011;118(9):2483-2491.

48. Ma G, Yasunaga J, Akari H, Matsuoka M. TCF1 and LEF1 act as T-cell intrinsic HTLV-1 antagonists by targeting Tax. Proc 
Natl Acad Sci USA. 2015;112(7):2216-2221.

49. Katayama S, et al. Antisense transcription in the mammalian transcriptome. Science. 2005;309(5740):1564-1566

50. Nevers A, et al. Antisense transcriptional interference mediates condition-specific gene repression in budding yeast. Nucleic Acids Res. 2018;46(12):6009-6025

51. Hobson DJ, Wei W, Steinmetz LM, Svejstrup JQ. RNA polymerase II collision interrupts convergent transcription. Mol Cell. 2012;48(3):365-374

52. Yoshida M, Satou Y, Yasunaga J, Fujisawa J, Matsuoka M. Transcriptional control of spliced and unspliced human T-cell leukemia virus type 1 bZIP factor (HBZ) gene. J Virol. 2008;82(19):9359-9368.

53. Melamed A, et al. The human leukemia virus HTLV-1 alters the structure and transcription of host chromatin in cis. Elife. 2018; 36245.

54. Nakano K, Watanabe T. HTLV-1 Rex Tunes the Cellular Environment Favorable for Viral Replication. Viruses. 2016;8(3):58

55. Armstrong AP, Franklin AA, Uittenbogaard MN, Giebler HA, Nyborg JK. Pleiotropic effect of the human T-cell leukemia virus Tax protein on the DNA binding activity of eukaryotic transcription factors. Proc Natl Acad Sci USA. 1993;90(15):7303-7307.

56. Suzuki T, Fujisawa JI, Toita M, Yoshida M. The trans-activator tax of human T-cell leukemia virus type 1 (HTLV-1) interacts with cAMP-responsive element (CRE) binding and CRE modulator proteins that bind to the 21-base-pair enhancer of HTLV-1. Proc Natl Acad Sci USA. 1993;90(2):610-614.

57. Baydoun HH, Bellon M, Nicot C. HTLV-1 Yin and Yang: Rex and p30 master regulators of viral mRNA trafficking. AIDS Rev. 2008;10(4):195-204

58. Nicot C, et al. HTLV-1-encoded p30II is a post-transcriptional negative regulator of viral replication. Nat Med. 2004;10(2):197-201.

59. Gaudray G, Gachon F, Basbous J, Biard-Piechaczyk M, Devaux C, Mesnard JM. The complementary strand of the human T-cell leukemia virus type 1 RNA genome encodes a bZIP transcription factor that down-regulates viral transcription. $J$ Virol. 2002;76(24):12813-12822.

60. Lemasson I, et al. Human T-cell leukemia virus type 1 (HTLV-1) bZIP protein interacts with the cellular transcription factor CREB to inhibit HTLV-1 transcription. J Virol. 2007;81(4):1543-1553.

61. Clerc I, et al. An interaction between the human T cell leukemia virus type 1 basic leucine zipper factor (HBZ) and the KIX domain of p300/CBP contributes to the down-regulation of tax-dependent viral transcription by HBZ. J Biol Chem 2008;283(35):23903-23913.

62. Philip S, Zahoor MA, Zhi H, Ho YK, Giam CZ. Regulation of human T-lymphotropic virus type I latency and reactivation by HBZ and Rex. PLoS Pathog. 2014;10(4):e1004040.

63. Jaenisch R, Bird A. Epigenetic regulation of gene expression: how the genome integrates intrinsic and environmental signals. Nat Genet. 2003;33 Suppl:245-254.

64. Feil R, Fraga MF. Epigenetics and the environment: emerging patterns and implications. Nat Rev Genet. 2012;13(2):97-109.

65. Cikos S, Bukovská A, Koppel J. Relative quantification of mRNA: comparison of methods currently used for real-time PCR data analysis. BMC Mol Biol. 2007;8:113. 